\title{
Endoscopic Transpapillary Gallbladder Drainage via the Mesh of Triple Uncovered Biliary Metal Stents Using a Soehendra Stent Retriever
}

\author{
Kazunari Nakahara, Yosuke Michikawa, Ryo Morita, Fumio Itoh \\ Department of Gastroenterology and Hepatology, St. Marianna University, School of Medicine, Kawasaki, Japan
}
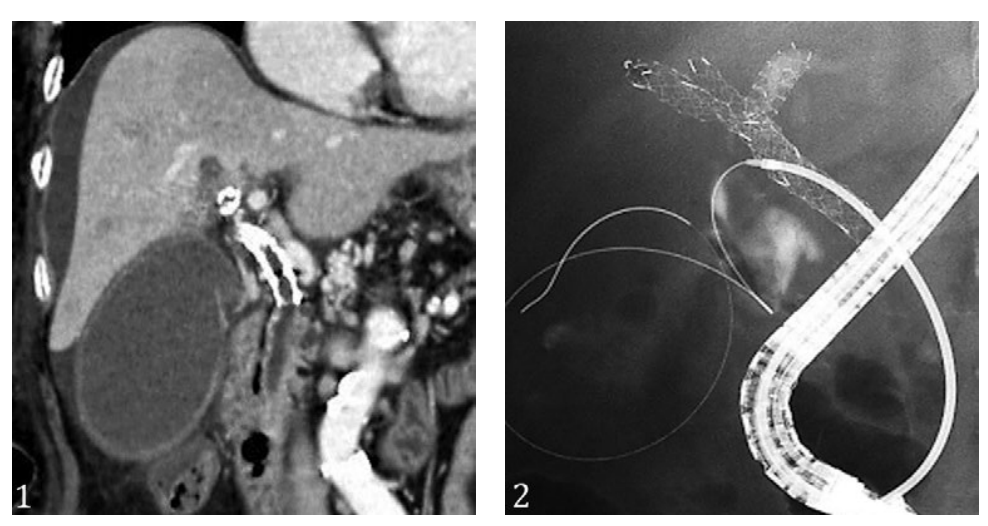

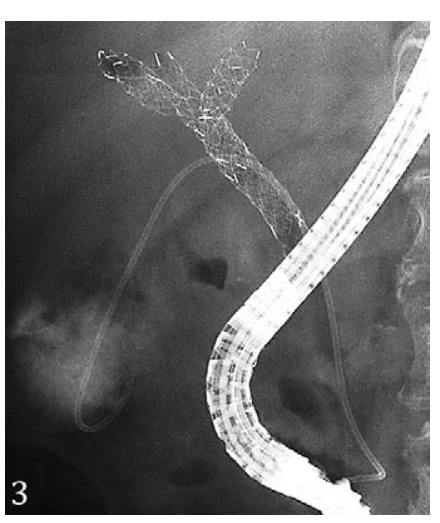

A 77-year-old woman with triple uncovered biliary selfexpandable metal stents (SEMSs) placed by partial stent-instent technique for hilar cholangiocarcinoma was admitted to our hospital owing to upper abdominal pain and high fever. Based on computed tomography findings, the patient was diagnosed with acute cholecystitis (AC) (Fig. 1). Because percutaneous transhepatic gallbladder drainage (PTGBD) was contraindicated due to ascites, endoscopic transpapillary gallbladder drainage (ETGBD) was planned. Cholangiography revealed that the triple SEMSs overlapped the orifice of the cystic duct (OCD). Hence, OCD was cannulated using a 0.035 inch hydrophilic guidewire, which was successfully advanced into the cystic duct via the mesh of the SEMSs. The guidewire, after insertion into the gallbladder, was changed to a stiff type. Next, we tried to dilate the mesh of the SEMSs using a tapered dilation catheter or a balloon catheter, but it could not be passed through the mesh. Therefore, we used a 7-Fr Soehendra stent retriever (Cook medical Inc., Bloomington, IN, United States) [1] successfully passing and dilating the mesh (Fig. 2). Finally, we were able to place a 5-Fr endoscopic naso-gallbladder tube via the mesh of the triple uncovered SEMSs (Fig. 3). No adverse events occurred, and AC immediately improved. Although the naso-gallbladder tube was self-removed 10 days after placement, there was no recurrence of AC until time of death.

To the best of our knowledge, this is a first report of a successful ETGBD performed via the mesh of previously placed triple biliary uncovered SEMSs that overlapped OCD. Although PTGBD has traditionally been the treatment of choice for AC after SEMS placement [2], ETGBD can be a treatment option, especially in the patients in whom PTGBD is contraindicated [3-5]. Using a Soehendra stent retriever may be useful for dilating the mesh of the SEMSs and facilitate the successful ETGBD.

Corresponding author: Kazunari Nakahara, nakahara@marianna-u.ac.jp

Conflicts of interest: None to declare.

\section{REFERENCES}

1. Soehendra N, Maydeo A, Eckmann B, Brückner M, Nam VC, Grimm $\mathrm{H}$. A new technique for replacing an obstructed biliary endoprosthesis. Endoscopy 1990;22:271-272. doi:10.1055/s-2007-1012868

2. Isayama H, Kawabe T, Nakai Y, et al. Cholecystitis after metallic stent placement in patients with malignant distal biliary obstruction. Clin Gastroenterol Hepatol 2006;4:1148-1153. doi:10.1016/j.cgh.2006.06.004

3. Itoi T, Sofuni A, Itokawa F, et al. Endoscopic transpapillary gallbladder drainage in patients with acute cholecystitis in whom percutaneous transhepatic approach is contraindicated or anatomically impossible (with video). Gastrointest Endosc 2008;68:455-460. doi:10.1016/j. gie.2008.02.052

4. Mori Y, Itoi T, Baron TH, et al. Tokyo Guidelines 2018: management strategies for gallbladder drainage in patients with acute cholecystitis (with videos). J Hepatobiliary Pancreat Sci 2018;25:87-95. doi:10.1002/ jhbp. 504

5. Nakahara K, Michikawa Y, Morita R, et al. Endoscopic transpapillary gallbladder stenting using a newly designed plastic stent for acute cholecystitis. Endosc Int Open 2019;7:E1105-E1114. doi:10.1055/a-0747-5668 